\title{
OPTIMISATION DES PROCESSUS DE FERMENTATION EN CONTINU
}

\author{
J. BLUM \\ Laboratoire d'Analyse Numërique \\ C.N.R.S et PARIS VI \\ 4. Place Jussieu \\ 75005 - PARIS - FRANCE
}

Abstract.

The knowledge of a mathematical model of the kinetics of growth of single cell microorganisms and of the physical system enabling to cultivate them leads to the mathematical formulation of the problem.

Duality and gradient methods have been used to realize the minimisation of the industrial cost of the process, the production of biomasse per hour being given. Because of non linearity of the state equations and of non convexity of the cost function, Uzawa and Arrow-Hurwicz algorithms have been improved so that the convergence is obtained in $30 \mathrm{sec}$. on Univac 1110 .

Ce travail entre dans le cadre de la préparation d'un Doctorat d'Etat ès Sciences Mathêmatiques à l'Université Paris VI. Je remercie le Professeur LIONS de m'avoir permis d'entreprendre ce travail, le Professeur KERNEVEZ de m'avoir mis en contact avec I'équipe de microbiologistes de Dijon, et le Professeur GLOWINSKI pour ses nombreux et judicieux conseils. Je remercie également toute l'équipe de la Station de Génie Microbiologique de l'INRA de Dijon, et en particulier MM. PERINGER et BLACHERE, pour leur fructueuse collaboration. 


\section{INTRODUCTION}

De façon très génêrale, on entend par "fermentation" la production en culture de microorganismes unicellulaires à partir de matières premières appelées substrats (sucres, mélasses,...) et à l'aide d'un agent fermenteur.

On distingue trois types de telles cultures :

- la culture batch : elle se fait à l'intêrieur d'un fementeur sans adjonction ni prélèvement d'un produit, jusqu'à épuisement du substrat. Ce type de culture est utilisé pour la fabrication d'antibiotiques et de vitamines.

- la culture en continu : on injecte du substrat de façon continue et on soutire ce qui se trouve dans le fermenteur de façon à ce que le volume reste constant. Ce type de culture est récent, encore peu industrialisê, mais utilisé pour obtenir les levures fourragères servant à l'alimentation animale.

- la culture en semi-continu : c'est un procédé mixte. On laisse la fermentation se faire en batch puis, au bout d'un certain temps, on injecte du substrat avec un débit variable au cours du temps. Il se produit une expansion de volume; quand le volume du liquide atteint celui du fermenteur, on vidange et on recommence. Ce procédé est utilisê pour la fabrication de la levure de boulangerie.

On se propose ici de réaliser une optimisation de la production de microorganismes unicellulaires en continu. La résolution de ce problème nécessite la connaissance du comportement du système biologique constitué par ces microorganismes ainsi que celle du système phỳsique permettant de les cultiver.

La modêlisation du système biologique a êté étudiêe en culture batch par 1 'équipe de la Station de Génie Microbiologique de l'I.N.R.A de Dijon [1] [2], et gếnéralisée au cas de la culture en continu [3]. La modélisation du système physique est celle présentée par Aiba, Humphrey et Millis dans [4]. Le problème consiste alors à minimiser le coût industriel du process, dépendant des paramètres biologiques et physiques.

Deux schémas de fermentation ont été étudiès; le premier est un schéma à un seul fermenteur standard avec recyclage, le second à deux fermenteurs couplés et avec recyclage. Les techniques employées sont prêsentées dans le cas le plus simple du schéma à un seul fermenteur. 


\section{I - MODELISATION.}

\section{1) Flow-sheet général.}

Ce flow-sheet simplifié rêsume bien la rêalité industrielle des fermentations en continu.

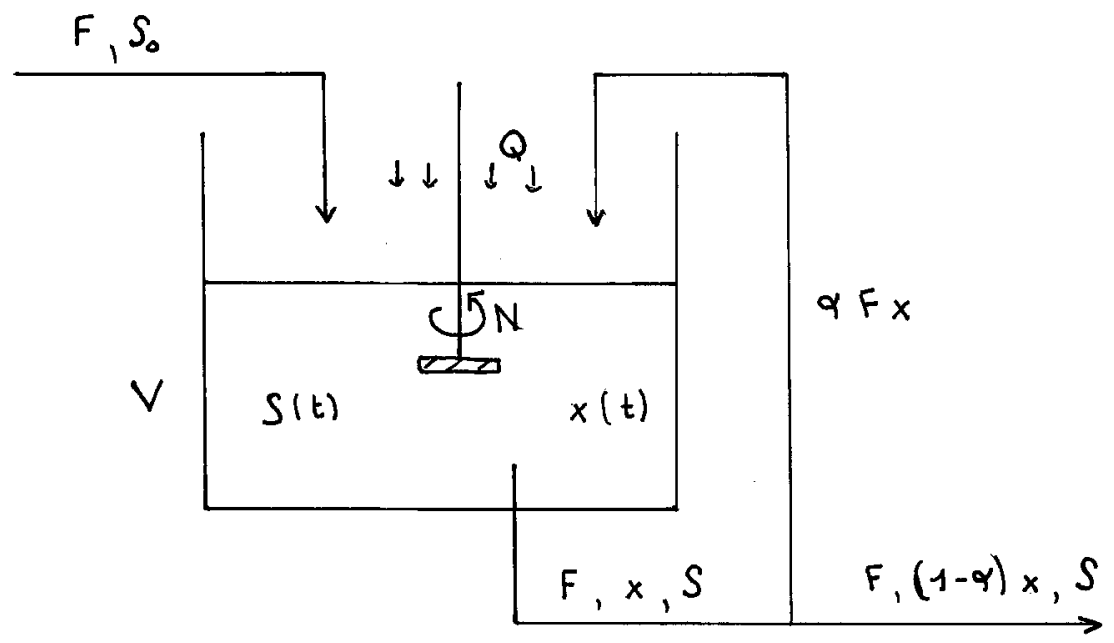

Fig. 1 Fermenteur standard avec recyclage, aération et agitation.

On injecte dans le fermenteur de volume utile $V$ du substrat de concentration $S_{0}$ avec un débit $F$ constant au cours du temps. A chaque instant la concentration du substrat à l'intërieux du fermenteur est $S(t)$ et celle de la biomasse produite est $x(t)$. On soutire du fermenteur de façon continue le milieu de culture avec le même débit $F$ de sorte que le volume reste constant. On recycle avec le coefficient de recyclage $\alpha$ une fraction de la biomasse soutirée et on recueille l'autre fraction ainsi que le substrat résiduel. De plus on injecte de l'air avec un débit $Q$ constant et on agite le milieu, la vitesse de rotation de l'agitateur étant $\mathbb{N}$.

- Le bilan en masse pour ce qui est de la biomasse s'écrit :

$$
V \frac{d x}{d t}=\mu x V-F(1-\alpha) x
$$

$\mu$ étant le taux de croissance spécifique de la biomasse.

- Le bilan en masse pour ce qui est du substrat s'écrit :

$$
\mathrm{V} \frac{\mathrm{dS}}{\mathrm{dt}}=\mathrm{F}\left(\mathrm{S}_{\mathrm{o}}-\mathrm{S}\right)-\mathrm{V}_{\mathrm{S}} \mathrm{xV}
$$

$\mathrm{V}_{\mathrm{S}}$ étant la vitesse spécifique d'absorption du substrat.

- Le développement des microorganismes nécessite la consommation de l'oxygène dissous 
dans le milieu de culture. Cet oxygène dissous, de concentration $\mathrm{C}_{\mathrm{L}}$, est transféré de la phase gazeuse dans la phase liquide par l'intermédiaire de l'aératjon et de 1 'agitation de la culture. Le bilan d'oxygène s'écrit :

$$
\frac{d C_{L}}{d t}=T_{R} \times K_{L A} \times\left(100-C_{L}\right)-\left(V_{R}+V_{R E}\right) x
$$

$\mathrm{K}_{\mathrm{LA}}$ étant le coefficient de transfert du système, $\mathrm{V}_{\mathrm{R}}$ la vitesse spécifique de respiration des microorganismes, $V_{R E}$ la vitesse spécifique de respiration endogène et $T_{R}$ une constante.

- A 1 'équilibre on aura donc :

(4) $\quad \mu \nabla=F(1-\alpha)$

(5) $\quad F\left(S_{0}-S\right)=V_{S} x V$

(6)

$$
\mathrm{T}_{\mathrm{R}} \times \mathrm{K}_{\mathrm{LA}} \times\left(100-\mathrm{C}_{\mathrm{L}}\right)=\left(\mathrm{V}_{\mathrm{R}}+\mathrm{V}_{\mathrm{RE}}\right) \mathrm{x}
$$

- La quantité de biomasse recueillie à l'êquilibre par unité de temps est

$$
\text { PROD }=F(1-\alpha) x
$$

\section{2) Modêlisation du système biologique}

\section{$\left[\begin{array}{lll}1 & ] & 2\end{array}\right][3]$}

Le taux de croissance spécifique $\mu$ de la biomasse, qui caractêrise le système biologique, est une combinaison linéaire de $V_{S}$ et de $V_{R}$ :

$$
\mu=P_{0} V_{S}+Q_{0} V_{R}
$$

Le système biologique est donc défini par la façon dont $V_{S}$ et $V_{R}$ dépendent de $\mathrm{S}$ et de la concentration d'oxygène dissous $\mathrm{C}_{\mathrm{L}}$. On a :

$$
V_{S}=V_{S M} \times \frac{S}{K_{S}+S} \times \frac{1}{1+\frac{B E \times C_{L}}{K_{B}+C_{I}}}
$$

où $V_{S M}$ est la vitesse spécifique maximale d'absorption du substrat, $K_{S}$, $K_{B}$ et $B E$ étant des constantes.

$$
V_{R}=\frac{S \times\left(K_{2} V_{1}+V_{2} s\right)}{K_{1} K_{2}+K_{2}\left(1+\frac{1}{K_{3}}\right) S+W s^{2}} \times \frac{C_{L}}{K_{L}+C_{L}}
$$

$\mathrm{V}_{1}, \mathrm{~V}_{2}, \mathrm{~K}_{1}, \mathrm{~K}_{2}, \mathrm{~K}_{3}, \mathrm{~K}_{\mathrm{L}}, \mathrm{W}$ étant des constantes.

De plus, la vitesse spécifique $V_{R E}$ de la respiration endogène est donnée par 1 'expression suivante :

$$
v_{R E}=v_{R E M} \times \frac{C_{L}}{K_{L E}+C_{L}}
$$


$V_{\text {REM }}$ étant sa valeur maximale et $K_{L E}$ une constante.

3) Modélisation du système physique [ 4 ]

Le système physique englobe la description de la géométrie du fermenteur et les divers processus de transfert de masse et d'énergie.

- La hauteur $H_{L}$ du liquide dans le fermenteur cylindrique étant choisie égale au diamètre de celui-ci, le volume utile du fermenteur est donné par :

$$
V=\frac{\pi}{4} \quad H_{L}^{3}
$$

- L'aération du fermenteur est caractérisée par un indice NAE appelé nombre d'aêration. Cet indice est lié au débit d'air injectê $Q$ et à la vitesse de rotation de l'agitateur $\mathrm{N}$ par la relation :

$$
\mathrm{NAE}=\alpha_{1} \frac{Q}{\mathrm{~N} \times \mathrm{H}_{\mathrm{L}}^{3}}
$$

- Soient PU la puissance absorbée par 1'agitation en absence d'aération et PG celle absorbée par l'agitation en présence d'aération. PU est donnée par :

$$
\mathrm{PU}=\beta_{1} \mathrm{~N}^{3} \mathrm{H}_{\mathrm{L}}^{5} \text { où } \beta_{1} \text { est une constante. }
$$

La courbe domant $\frac{\mathrm{PG}}{\mathrm{PU}}$ en fonction de $\mathrm{NAE}$ est une relation empirique :

$$
P G=P J \times h(N A E)
$$

- La vitesse linéaire $V_{L}$ de l'air traversant la section principale du fermenteur et ramenée à cette section vaut :

$$
\mathrm{V}_{\mathrm{L}}=\frac{\mathrm{Q}}{\pi / 4 \mathrm{H}_{\mathrm{L}}^{2}}
$$

- Le coefficient de transfert $\mathrm{K}_{\mathrm{LA}}$ dépend de la géométrie du fermenteur; de la pression PR de l'air à l'intérieur du fermenteur (exprimée en mm Hg), de la puissance d'agitation $P G$ et de la vitesse de l'air $V_{L}$ par la relation :

$$
\mathrm{K}_{\mathrm{LA}}=\delta_{1} \times\left[2 \times\left(1+\frac{\mathrm{PR}-760}{760}\right)+\frac{\mathrm{H}_{\mathrm{L}}}{10,3}\right] \times\left(\frac{\mathrm{PG}}{\mathrm{V}}\right)^{0,95} \times \mathrm{v}_{\mathrm{L}}^{0,67}
$$

où $\delta_{1}$ dépend de $P R$ et de la température $T$ de fermentation.

- D'une aération intense rêsulte une augmentation apparente du volume utile du fermenteur qui, exprimé en $0 / 0$, est appelée hold-up HOL et est donnée par la relation :

$$
\text { HOL }=\left[\left(\frac{\mathrm{PU}}{\mathrm{V}}\right)^{0,4} \times \mathrm{V}_{\mathrm{L}}^{0,5}-2,75\right] 10,725
$$

Le volume total du fermenteur, compte-tenu d'une formation éventuelle de mousse $V_{0}$ évaluée à $0,2 \mathrm{~V}$, vaut alors :

$$
\mathrm{V}_{\mathrm{T}}=\mathrm{V}+\mathrm{V} \times \frac{\mathrm{HOL}}{100}+\mathrm{V}_{\mathrm{O}}=\mathrm{V} \times\left(1,2+\frac{\mathrm{HOL}}{100}\right)
$$


Pour optimiser le process le biologiste a la possibilité de jouer sur un certain nombre de paramètres qui, une fois fixés, déterminent totalement le système : ce sont les variables de contrôle du système.

On prendra pour vecteur de contrôle:

$$
u=\left(F, s_{0}, \alpha, H_{L}, N A E, C_{L}\right) \in R^{6}
$$

Le vecteur d'état $y$ est alors :

$$
y=\left(S, x, K_{L A}, N, Q, P U, P G, V_{L}, H O L, V_{T}\right) \in R^{10}
$$

1) Equations d'état

A l'équilibre, y est dêterminé à partir de u par les êquations implicites (4), (5), (6) et (13) à (19). Ce système sera notê :

$$
A(u, y)=0
$$

où A est non linéaire.

\section{2) Contraintes}

a) Contraintes sur le contrôle :

Les variables de contrôle sont soumises aux contraintes suivantes :

$$
\begin{aligned}
& 0<F \leqslant F \max \\
& 0<S_{0} \leqslant S_{0} \max \\
& 0 \leqslant \alpha<1 \\
& 0<F_{L} \leqslant H_{L} \max \\
& 0<N A E \leqslant 12 \\
& 1<C_{L}<100
\end{aligned}
$$

On transformera les inégalités strictes en inégalitês larges : par exemple $F>0$ sera remplacé par $F \geqslant F_{\min }$ où $F_{\min }$ est suffisamment voisin de 0 pour que la signification physique de la contrainte reste la même. On peut alors écrire : $u \in K_{0}$, où $K_{0}$ est un convexe fermé borné de $R^{6}$.

\section{b) Contraintes sur l'état :}

Pour que le modèle du I soit valable il nous faut imposer les contraintes suivantes :

$$
\begin{aligned}
& \mathrm{x} \leqslant 50 \mathrm{~g} / 1 \\
& \mathrm{~V}_{\mathrm{L}} \leqslant 150 \mathrm{~m} / \mathrm{h} \\
& 0,1 \mathrm{HP} / \mathrm{m}^{3} \leqslant \frac{\mathrm{P}_{\mathrm{G}}}{\mathrm{V}} \leqslant 2,5 \mathrm{HP} / \mathrm{m}^{3} \rho \text { étant la densité du milieu, VIS sa viscosité, cette } \\
& \frac{\mathrm{N} \times \mathrm{H}_{\mathrm{I}}^{2} \times \rho}{9 \times \mathrm{VIS}} \geqslant 6000 \quad \begin{array}{l}
\text { contrainte signifie que le nombre de Reynolds est } \\
\text { suffisamment grand pour qu'on soit en régime turbulent. }
\end{array}
\end{aligned}
$$


On notera ces contraintes sous la forme : $f(u, y) \leqslant 0$ où $f \in R^{5}$

c) Contrainte "spéciale"

Lorsque u est fixée, pour résoudre le système des équations d'état, on utilise (4) :

$$
\mu\left(S, C_{L}\right)=\frac{F(1-\alpha)}{\pi / 4 H_{L}^{3}}
$$

Mais on doit avoir $S<S_{0}$ pour assurer la positivité de $x$ et des autres vaiables d'état ; $\mu$ étant strictement croissante par rapport à $S$, on doit avoir :

$$
F(1-\alpha)-\pi / 4 H_{L}^{3} \mu\left(S_{0}, C_{L}\right)<0
$$

qu'on note $g(u)<0$.

\section{3) Fonction-coût :}

Le coût global du process est composé par les coûts unitaires d'investissement et de fonctionnement des postes qui le composent : production (PROD), matières premières (FS ${ }_{0}$ ), agitation ( $\left.P G\right)$, aération ( $\left.Q\right)$, épuration (FS), écoulement (F), recyclage (RE), fermenteur $\left(V_{T}\right)$. I1 s'écrit :

$$
\begin{aligned}
\text { COUT } & =Q_{1} P R O D+Q_{2} F S+Q_{3} F_{0}+Q_{4} P^{E_{1}}+Q_{5} Q^{E_{2}}+Q_{6} F+Q_{7} V_{T}+Q_{8} V_{T}^{E^{3}}+Q_{9} P_{G}^{E_{4}} \\
& +Q_{10} Q^{E_{5}}+Q_{11} R E+Q_{12} P R O D+Q_{13}
\end{aligned}
$$

le coefficient de recyclage $\mathrm{RE}$ ètant donnê par :

$$
\begin{array}{ll}
\mathrm{RE}=0 & \text { si } \alpha=0 \text { (pas de recyclage) } \\
\mathrm{RE}=1 & \text { si } \alpha>0 \text { (nëcessité d'un système de recyclage) }
\end{array}
$$

On désire également que la production de biomasse PROD donnée par (7) soit proche d'une valeur $K$ qui représente la production désirée de biomasse.

On prendra alors pour critère à minimiser une somme pondérée de coUT et de $(\mathrm{PROD}-\mathrm{K})^{2}$ :

$$
J=C O U T+C(P R O D-K)^{2}
$$

$\mathrm{C}$ étant la constante permettant au biologiste de donner plus ou moins d'importance

à la contrainte sur la production.

COUT contient un terme non différentiable $Q_{11} R E$, qui est une fonction en escalier. $J$ peut donc se mettre sous la forme : $\mathrm{J}=\mathrm{J}_{0}+\mathrm{J}_{1}$ où $J_{0}$ est diffërentiable et où $J_{l}$ est une fonction en escalier.

4) Formulation du problème d'optimisation:

Existe-t-il ( $\left.u_{0}, y_{o}\right) \in K \times R^{10}$ tel que :

$$
\begin{array}{ll}
A\left(u_{0}, y_{0}\right)=0, \quad g\left(u_{0}\right)<0 . & f\left(u_{0}, y_{0}\right) \leqslant 0 \\
\text { et } & J\left(u_{0}, y_{0}\right)=\quad \text { inf } J(u, y) \\
& \\
& y \in R R^{10} \\
& A(u, y)=0 \\
& g(u)<0 \\
& f(u, y) \leqslant 0
\end{array}
$$




\section{II - RESOLUTION DU PROBLEME D'OPTIMISATION (P)}

On démontre aisêment à $l^{\prime}$ aide du théorème des fonctions implicites et par des arguments de compacité que (P) admet au moins une solution.

A étant non linéaire et $J$ non convexe il y a tout lieu de penser qu'il existe plusieurs minima locaux. Ce sont ces minima qu'on va chercher à atteindre.

Soit $K^{\prime}=\left\{u \in K_{0}: g(u)<0\right\}$

$g$ étant non convexe, $\mathrm{K}^{\prime} \mathrm{n}^{\prime}$ est pas convexe.

Le problème de recherche des optima locaux se pose alors ainsi: Existent-ils $\left(u_{0}, y_{0}\right) \in K^{\prime} \times R^{10}$ et un voisinage $V^{\prime}$ de $u_{0}$ dans $R^{6}$ tels que

et

$$
A\left(u_{0}, y_{0}\right)=0, f\left(u_{0}, y_{0}\right) \leqslant 0
$$

$$
\begin{aligned}
J\left(u_{0}, y_{0}\right)= & \inf J(u, y) \\
& u \in V^{\prime} \cap K^{\prime} \\
& y \in R^{10} \\
& A(u, y)=0 \\
& f(u, y) \leqslant 0
\end{aligned}
$$

\section{Principe de la méthode :}

L'idée est de résoudre ( $\left.P^{\prime}\right)$ par une méthode de type gradient, en traitant la contrainte $u \in K$ par projection, $g(u)<0$ pax continuité, $f(u, y) \leqslant 0$ à $1^{\prime}$ aide de multiplicateurs de Lagrange et $A(u, y)=0$ par l'introduction d'un état adjoint.

1) Recherche d'un point-selle pour résoudre la contrainte $f(u, y) \leqslant 0$

Pour traiter la contrainte $f(u, y) \leqslant 0$, on introduit le lagrangien

$$
\mathcal{L}(u, y, q)=J(u, y)+(q, f(u, y)) \quad, \quad q \in R_{+}^{5}
$$

$\mathcal{L}$ peut se mettre sous la forme :

$$
\mathcal{L}=\mathcal{L}_{0}+Q_{11} R E=\mathcal{L}_{0}+\mathcal{L}_{1}
$$

où $\mathscr{L}_{\text {o }}$ est différentiable et $\mathscr{L}_{1}$ une fonction en escalier.

$q_{0}$ est vecteur de Kuhn-Tucker $[5]$ pour ( $\left.{ }^{\prime}\right)$ si:

$$
\begin{array}{ll}
\inf \mathcal{L}\left(\mathrm{u}, \mathrm{y}, \mathrm{q}_{0}\right)= & \inf \mathrm{in}(\mathrm{u}, \mathrm{y}) \\
\mathrm{u} \in \mathrm{V}^{\prime} \cap \mathrm{K}^{3} & \mathrm{u} \in \mathrm{V}^{\prime} \cap \mathrm{K}^{1} \\
\mathrm{y} \in \mathrm{R}^{10} & \mathrm{y} \in \mathrm{R}^{10} \\
\mathrm{~A}(\mathrm{u}, \mathrm{y})=0 & \mathrm{~A}(\mathrm{u}, \mathrm{y})=0 \\
& \mathrm{f}(\mathrm{u}, \mathrm{y}) \leqslant 0
\end{array}
$$

On sait que, pour que $u_{0}$ et $y_{0}$ soient solutions de $\left(P^{\prime}\right)$ et pour que q soit vecteur de Kuhn-Tucker associé, il faut et il suffit que $\left(\left(u_{o}, y_{0}\right), q_{o}\right)$ soit point-selle de $\&$ sur $\left(V^{\prime} \cap K^{\prime}\right) \times R^{10} \times R_{+}^{5}$.

Du fait de la non-convexité, l'existence de point-selle n'est pas assuré, mais pour trouver $u_{0}$ et $y_{o}$ on emploiera malgré tout une technique de recherche de point-selle. $[6]$ [ 7 ]

a) Algorithme $\left(A_{,}\right)$de type Uzawa

On part de $\mathrm{u}_{1} \in \mathrm{K}^{\mathrm{I}}, \mathrm{q}_{1} \in \mathrm{R}_{+}^{5}$.

A la $n^{e}$ itération, on calcule $u_{n}, y_{n}$ et $q_{n}$ de la façon suivante: 
$\left(a_{1}\right)\left\{\begin{array}{l}u_{n} \in V^{\prime} \cap k_{n} \cap K^{\prime} \\ A\left(u_{n}, y_{n}\right)=0 \\ \mathcal{L}\left(u_{n}, y_{n}, q_{n-1}\right)=\quad \text { inf } \quad \mathcal{L}\left(u, y, q_{n-1}\right)\end{array}\right.$

$$
\begin{aligned}
& u \in V^{\prime} \cap K_{n} \cap K^{\prime} \\
& y \in \in R^{10} \\
& A(u, y)=0
\end{aligned}
$$

$q_{n}=\operatorname{Proj} R_{+}^{5}\left(q_{n-1}+\rho^{\prime} f\left(u_{n}, y_{n}\right)\right) \quad \rho^{\prime}>0$

Choix de $\mathrm{K}_{\mathrm{n}}$ :

Soit $\mathrm{k}$ un réel positif compris entre 0 et 1 .

Soit $\mathscr{H}_{n} l$ 'homothétie de centre $u_{n}$ et de rapport $k$.

$\mathrm{K}_{\mathrm{n}}$ est 1 'homothétique de $\mathrm{K}_{0}$ par la transformation $\mathcal{H}_{\mathrm{n}}$.

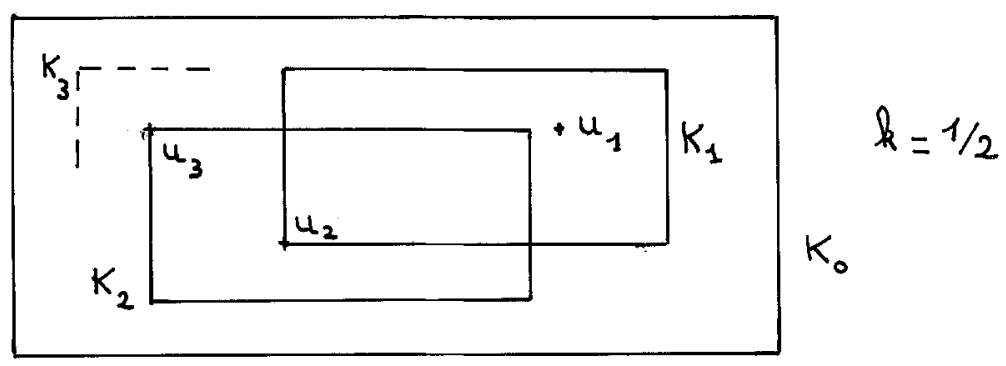

Fig. 2 : Construction de $\mathrm{K}_{\mathrm{n}}$ (représentation dans $\mathrm{R}^{2}$ )

A chaque itération de cet algorithme, la recherche de $u_{n}$ et de $y_{n}$ nous amène à résoudre le problème de contrôle optimal $\left(a_{1}\right)$ qu'on traitera plus bas.

I1 convient également de remarquer que, si cet algorithme converge, ce sera vers un point maximin de $\mathcal{L}$ et non nécessairement vers un point-selle. Il faudra donc s'assurer que $u_{0}$ et $y_{0}$ sont bien solutions de ( $\left.P^{\prime}\right)$.

b) Algorithme $\left(\mathrm{A}_{2}\right)$ de type Arrow-Hurwicz :

Du système des équations d'état $A(u, y)=0$, on peut, come $\frac{\partial A}{\partial y}$ est inversible, exprimer en thêorie, $y$ en fonction de $u$ : $y=\bar{y}(u)$.

$D^{\prime}$ où $\quad \mathcal{L}_{0}(\mathrm{u}, \mathrm{y}, \mathrm{q})=\mathcal{L}_{0}(\mathrm{u}, \overline{\mathrm{y}}(\mathrm{u}), \mathrm{q})=\overline{\mathcal{L}}_{0}(\mathrm{u}, \mathrm{q})$

L'algorithme $\left(A_{2}\right)$ peut alors s'écrire ainsi :

- On part de $\mathrm{u}_{1} \in \mathrm{K}^{\prime}, \mathrm{q}_{1} \in \mathrm{R}_{+}^{5}$.

- A la $n$ itération, on calcule $u_{n}$, $y_{n}$ et $q_{n}$ de façon suivante :

$$
\left\{\begin{array}{l}
u_{n}=\operatorname{Proj}_{K_{n}}\left(u_{n-1}-\rho_{n} \frac{t_{\partial \bar{f}_{O}}}{\partial u}\left(u_{n-1}, q_{n-1}\right)\right) \\
A\left(u_{n}, y_{n}\right)=0 \\
q_{n}=\operatorname{Proj}_{R_{+}^{5}}\left(q_{n-1}+\rho^{\prime} f\left(u_{n}, y_{n}\right)\right), p^{\prime}>0
\end{array}\right.
$$

$K_{n}$ est défini de la même façon que dans $\left(A_{1}\right)$ et $p_{n}$ sera précisé plus tarả. Si 
cet algorithme converge, il faudra également s'assurer que $u_{0}$ et $y_{0}$ sont solutions de $\left(\mathrm{P}^{i}\right)$.

2) Résolution du problème de contrôle $\left(a_{1}\right)$ et calcul de $\frac{\partial \overline{\mathcal{L}}_{0}}{\partial \mathrm{u}}$ :

L'idêe, pour rêsoudre $\left(a_{1}\right)$, est de traiter les équations d'état sous forme de contraintes [8] [ 9 ] et donc d'introduire le lagrangien:

$$
M_{n-1}(u, y, p)=J(u, y)+\left(q_{n-1}, f(u, y)\right)+(p, A(u, y)) p \in R^{10}
$$

On démontre aisêment le théorème suivant :

THEOREME Pour que $\left(u_{n}, y_{n}, p_{n}\right)$ soit point-selle de $M_{n-1}$ sur $\left(\left(V^{\prime} \cap K^{\prime} \cap K_{n}\right) \times R^{10}\right) \times R^{10}$, il faut que :

$$
A\left(u_{n}, y_{n}\right)=0
$$

$\frac{t^{\prime} J_{0}}{\partial y}\left(u_{n}, y_{i}\right)+\frac{t}{\partial y}\left(u_{n}, y_{n}\right) q_{n-1}+\frac{\partial A}{\partial y}\left(u_{n}, y_{n}\right) p_{n}=0$

$\left(\frac{t}{\partial u} \overline{\mathcal{L}}_{0}\left(u_{n}, q_{n-1}\right), u-u_{n}\right)_{R^{6}}+\mathcal{L}_{1}(u)-\mathcal{L}_{1}\left(u_{n}\right) \geqslant 0 \quad \forall u \in V^{\prime} \cap K_{n} \cap k^{\prime}$

avec :

$$
\frac{t_{\partial \bar{L}_{0}}}{\partial u}\left(u_{n}, q_{n-1}\right)=\frac{t J_{O}}{\partial \bar{u}}\left(u_{n}, y_{n}\right)+\frac{t_{\partial f}}{\partial u}\left(u_{n}, y_{n}\right) q_{n-1}+\frac{t A}{\partial u}\left(u_{n}, y_{n}\right) p_{n}
$$

Pour résoudre le problème de contrôle $\left(a_{1}\right)$ on pourra alors utiliser 1'algorithme de type gradient projeté suivant:[10] [11]

- On part de $u_{n}^{0}=u_{n-1}$

- La ie itération donnant $y_{n}^{i}, p_{n}^{i}$ et $u_{n}^{i+1}$ à partir de $u_{n}^{i}$ s'écrit :

$$
\begin{aligned}
& A\left(u_{n}^{i}, y_{n}^{i}\right)=0 \\
& {\frac{t}{\partial J_{0}}}_{\partial y}\left(u_{n}^{i}, y_{n}^{i}\right)+\frac{t_{\partial f}}{\partial y}\left(u_{n}^{i}, y_{n}^{i}\right) q_{n-1}+\frac{t A}{\partial y}\left(u_{n}^{i}, y_{n}^{i}\right) p_{n}^{i}=0 \\
& u_{n}^{i+1}=\operatorname{Proj}_{K_{n}}\left(u_{n}^{i}-p_{n}^{i}\left(\frac{\partial J_{0}}{\partial u}\left(u_{n}^{i}, y_{n}^{i}\right)+\frac{t f}{\partial u}\left(u_{n}^{i}, y_{n}^{i}\right) q_{n-1}+\frac{\partial A}{\partial u}\left(u_{n}^{i}, y_{n}^{i}\right) p_{n}^{i}\right)\right)
\end{aligned}
$$

- $u_{n}^{\infty}=u_{n}$

$\frac{\text { Choix de } \rho_{n}^{i}}{\rho_{n}^{i} \text { sera }}$ converge vers un minimum local. Si on a par exemple une fonction $\mathcal{L}$ de ce type :

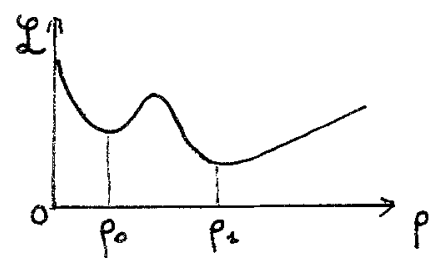


c'est $\rho_{0}$ qu'on retiendra. C'est dans ce sens que la minimisation de $\mathcal{L}$ est effectuée à I'intérieur d'un voisinage $V^{\prime}$ de uo comme cela a été défini dans l'algorithme $\left(A_{1}\right)$.

$p_{n}^{i}$ devra également être tel que $u_{n}^{i+l}$ appartienne à $K^{\prime}$.

- Pratiquement on déterminera d'abord $\rho_{n}^{i} \max$ tel que les $\rho$ de I'intervalle $\left.\left[0, p_{n}^{i}\right]_{\max }\right]$ soient en bijection avec les points du projeté sur $\mathbb{K}_{n}$ de la demi-droite de descente d'origine $u_{n}^{i}$.

Dans la recherche du $\rho$ optimal, il n'est pas judicieux de procéder par dichotomie du fait du caractère non convexe de $\mathcal{L}$. On divisera les intervalles en dix intervalles égaux, ce qui permet de mieux suivre I'évolution de $\mathcal{L}$. en fonction de $\rho$.

On calculera $\mathcal{L}\left(\operatorname{Proj}_{K_{n}}\left(u_{n}^{i}-\frac{j}{10} \rho_{n \max }^{i} \frac{t_{\partial} \vec{L}_{o}}{\partial u}\left(u_{n}^{i}, q_{n-1}\right)\right)\right)$

et

$$
g\left(\operatorname{Proj}_{K}\left(u_{n}^{i}-\frac{j}{10} \rho_{n \max }^{i} \frac{t_{\partial \bar{L}_{o}}}{\partial u}\left(u_{n}^{i}, q_{n-1}\right)\right)\right)
$$

pour $j$ allant de 1 à 10 et dès que $\&$ ne décrôit plus ou que $g$ devient positif (pour un certain $j_{0}$ ), on recommence I'opération sur l'intervalle

$I=\left[u_{n}^{i}-\frac{j_{0}^{-2}}{10} \rho_{n \max }^{i} \frac{t_{\partial \bar{L}_{o}}}{\partial u}, u_{n}^{i}-\frac{j_{o}}{10} \rho_{n}^{i} \max \frac{t_{\partial \bar{L}_{o}}}{\partial u}\right]$

(si $j_{0} \neq 1$ ) jusqu'à ce que $\mathcal{L}$ soit minimum avec la précision qu'on souhaite. On est amené à considérer l'intervalle I ci-dessus car on peut avoir les deux configurations suivantes :

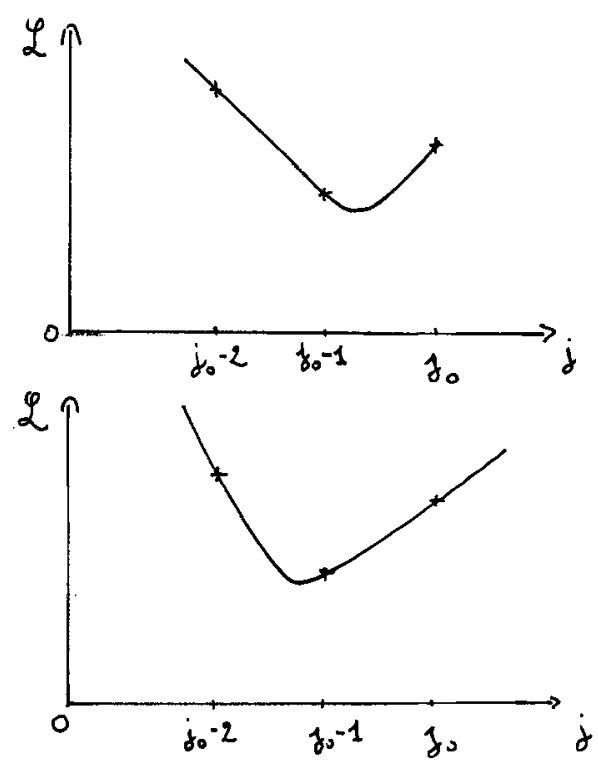


- On constate que, $\mathrm{si}$ on a utilisé le gradient de $\mathfrak{L}_{0}$ dans $I^{\prime}$ algorithme, $c^{\prime}$ est

$\mathcal{L}$ qu'on minimise par 1 'intermédiaire de $\rho_{n}^{i}$ en accord avec (20) ou $\left(\mathcal{L}_{1}(\mathrm{u})-\mathcal{L}_{1}\left(\mathrm{u}_{n}\right)\right)$ vaut soit 0 soit $Q_{11}$.

- $\rho_{n}^{i}$ est donc tel que $u_{n}^{i+1}$ soit le premier minimum local de $\mathcal{L}$ à partir de $u_{n}^{i}$ sur le projeté sur $K_{n}$ de la demi-droite de descente à condition que g soit resté négatif ou à défaut est tel que $u_{n}^{i+1}$ annule $g$ pour la première fois sur le projeté de cette demi-droite. C'est donc le choix de $\rho_{n}^{i}$ qui permet de faire comme on $I^{\prime a}$ vu dans $l a$ définition de $\left(A_{1}\right)$ une minimisation de $\mathfrak{L}$ sur $V^{\prime} \cap K_{n} \cap K^{\prime}, V^{\prime}$ à cause du $\rho$ localement optimal, $K_{n}$ de la projection et $\mathrm{K}^{\prime}$ parce qu'on oblige $\mathrm{u}_{\mathrm{n}}^{\mathrm{i}}$ à rester dans $\mathrm{K}^{\prime}$.

3) Formes definitives des algorithmes $\left(A_{1}\right)$ et $\left(A_{2}\right)$ :

a) Algorithme $\left(A_{1}\right)$ :

- On part de $u_{1} \in K^{y,} q_{1}=0$

- On calcule $u_{n}$ à partir de $u_{n-1}$ et $q_{n-1}$ à l'aide de l'algorithme suivant : - $u_{n}^{o}=u_{n-1}$

- $1 a i^{e}$ itération donnant $u_{\pi}^{i+1}$ à partir de $u_{n}^{i}$ s'êcrit : $A\left(u_{n}^{i}, y_{n}^{i}\right)=0$ $\frac{t_{\partial} J_{0}}{\partial y}\left(u_{n}^{i}, y_{n}^{i}\right)+\frac{t}{\partial y}\left(u_{n}^{i}, y_{n}^{i}\right) q_{n-1}+\frac{t}{\partial y}\left(u_{n}^{i}, y_{n}^{i}\right) p_{n}^{i}=0$ $u_{n}^{i+1}=\operatorname{Proj} k_{n}\left[u_{n}^{i}-p_{n}^{i}\left(\frac{\partial J_{o}}{\partial u}\left(u_{n}^{i}, y_{n}^{i}\right)+\frac{\partial f}{\partial u}\left(u_{n}^{i}, y_{n}^{i}\right) q_{n-1}+\frac{\partial A}{\partial u}\left(u_{n}^{i}, y_{n}^{i}\right) p_{n}^{i}\right)\right]$ $\cdot u_{n}^{\infty}=u_{n}$

- $A\left(u_{n}, y_{n}\right)=0$

- $q_{n}=\operatorname{Proj}_{R_{+}^{5}}\left(q_{n-1}+\rho^{\prime} f\left(u_{n}, y_{n}\right)\right) \quad \rho^{\prime}>0$

b) Algorithme $\left(A_{2}\right)$ :

On part de $u_{1} \in K^{3}, q_{1}=0$

- ${ }_{n}$, $y_{n}$ et $q_{n}$ se calculent à partir de $u_{n-1}, y_{n-1}$ et $q_{n-1}$ de 1 a façon suivante :

$$
\begin{aligned}
& \frac{t_{\partial J}}{\partial y}\left(u_{n-1}, y_{n-1}\right)+\frac{\partial f}{\partial y}\left(u_{n-1}, y_{n-1}\right) q_{n-1}+\frac{\partial A}{\partial y}\left(u_{n-1}, y_{n-1}\right) p_{n-1}=0 \\
& u_{n}=\operatorname{Proj}_{k}\left(u_{n-1}-\rho_{n}\left(\frac{t_{0}}{\partial u}\left(u_{n-1}, y_{n-1}\right)+\frac{\partial f}{\partial u}\left(u_{n-1}, y_{n-1}\right) q_{n-1}+\frac{\partial A_{1}}{\partial u}\left(u_{n-1}, y_{n-1}\right) p_{n-1}\right)\right)
\end{aligned}
$$


$A\left(u_{n}, y_{n}\right)=0$

$q_{n}=\operatorname{Proj}_{R_{+}^{5}}\left(q_{n-1}+p^{\prime} f\left(u_{n}, y_{n}\right)\right) \quad \rho^{\prime}>0$

$\rho_{n}$ est choisi de la même façon que $\rho_{n}^{0}$ dans $\left(A_{1}\right)$.

Remarque :

Justification du traitement spécial de la contrainte $g(u)<0$

- Nous avons vu que cette contrainte équivaut à $S<S_{0}$. La contrainte $S<S_{0}$ aurait pu être incluse dans $f$ et traitée par dualitê, mais on constate que les algorithmes proposés nécessitent à chaque itération la résolution du système des équations d'état $A(u, y)=0$. Or si la contrainte $s<S_{0}$ était traitée à l'aide de multiplicateurs de Lagrange on pourrait avoir au cours de l'algorithme $S_{n}>S_{0}$ donc $x_{n}<0$ ce qui n'a plus de signification physique et empêche de résoudre le système des équations d'état.

- On aurait également pu penser traiter $g(u)<0$ par projection sur $K^{\prime}$ au lieu de projeter sur $K$, mais $K^{\prime} n^{\prime}$ est pas convexe et cette projection serait difficile.

- On a donc traité $g(u)<0$ par continuité ; $g$ étant continue, $g\left(u_{n}\right)$ négatif, il existe un voisinage de $u_{n}$ où $g$ est négatif et l'optimisation se fait dans ce voisinage. En fait cette contrainte ne peut devenir bloquante que dans les premières itérations car :

$g(u)=0 \Rightarrow x=0 \Rightarrow$ PROD $=0 \Rightarrow J=\operatorname{COUT}+\mathrm{CK}^{2}$

$\mathrm{J}$ est alors très grand et ne peut donc être un minimum. 


\section{IV - MISE EN OEUVRE - RESULTATS}

\section{1) Mise en peuvre}

Le choix de la valeur unitiale $u_{1}$ dans les algorithmes est important du fait de la convergence vers des minima locaux. On a effectué un maillage de $\mathrm{K}_{\mathrm{o}}$ et étudié $J$, f et g aux noeứs de ce maillage. Les algorithmes ont été mis en oeuvre à partir de valeurs $u_{1}$ telles que $J\left(u_{1}\right)$ soit "assez petit" et que les contraintes soient vérifiêes en ces points.

Rappelons que le lagrangien $\mathcal{L}$ s'écrit :

$$
\mathcal{L}(u, y, q)=J(u, y)+\sum_{j \in 1}^{5} q_{j} E_{j}(u, y)
$$

Pour assurer une bonne pondération dans le lagrangien entre $J$ et les termes $q_{j} f_{j}$, on a remplacé $f_{j}$ par $k_{j} f_{j}$ et choisi $k_{j}$ de sorte que $q_{j} f_{j}$ ait sensiblement comme ordre de grandeur le $1 / 10$ de la valeur de $\mathrm{J}$.

La constante $\rho^{\prime}$ des algorithmes (A1) et (A2) a été prise égale à 1. On a adopté pour test d'arrêt des algorithmes l'optimalité de $J$ à $10^{-5}$ près et la nullité de $q_{j} f_{j}$ pour $j \in\{l, \ldots, 5\}$. Les valeurs numëriques adoptées sont celles de la cinétique du Saccharomyces Cerivisiae.

2) Importance du rapport d'homothetie k et comparaison de (A1) et (A2).

\begin{tabular}{|c|c|c|}
\hline$k$ & $A 1$ & $A 2$ \\
\hline $1 / 2$ & $\begin{array}{c}45 \text { iterations } \\
37 \mathrm{~s} \\
J_{\operatorname{fin}}=3560\end{array}$ & $\begin{array}{l}200 \text { itérations } \\
1 \text { mn } \\
J_{\text {fin }}=5085\end{array}$ \\
\hline 1 & $\begin{array}{c}200 \text { itérations } \\
1 \mathrm{mn} \\
J_{\text {fin }}=4800\end{array}$ & $\begin{array}{c}81 \text { itérations } \\
38 \mathrm{~s} \\
J_{\text {fin }}=3560\end{array}$ \\
\hline
\end{tabular}

Fig. 3 Comparaison de $\left(A_{1}\right)$ et $\left(A_{2}\right)$ suivant diverses valeurs de $k$ sur un exemple. 
Les essais numériques à partir de diverses valeurs initiales $u_{1}$ montrent que l'algorithme (A1) de type Uzawa, pris avec $k=1$, c'est à dire avec optimisation dans tout $K$, converge vers un optimum médiocre après de nombreuses itérations. La raison en est que $J$ est brutalement minimisế dans les premières itérations, que les contraintes deviennent alors fortement positives et que les multiplicateurs de Lagrange agissent alors si brutalement pour les ramener en-dessous de 0 que $\mathrm{J}$ atteint des valeurs très élevées, ce qui créê de très fortes oscillations de $J$ dans les premières itérations et entraine une convergence médiocre. C'est ce qui a conduit à utiliser une restriction homothétique et on constate en général que pour $k=1 / 2,1^{\prime a l g o r i t h m e ~(A l) ~ c o n v e r g e ~ r e ́ g u l i e ̀ r e m e n t ~ e t ~ v i t e ~ v e r s ~ d e ~ b o n s ~ o p t i m a ~ l o c a u x . ~}$

L'algorithme (A2) utilisé avec $k=1$ est meilleur que (A1) avec le même $k$, car on ne fait qu'une descente suivant le gradient à chaque itération et l'optimisation est donc moins forte, ne créant pas de brusques oscillations. Par contre pour $k$ plus petit 1'algorithme (A2) est en gënéral moins bon que (A1), car il converge plus lentement et même parfois vers de moins bons optima.

En définitive il semble que la meilleure méthode soit d'adopter (Al) avec $k=1 / 2$ et, si on $n$ 'a pas une bonne et rapide convergence, soit de diminuer $k$ en utilisant toujours (A1), soit d'utiliser (A2) mais en augmentant $k$.

\section{REMARQUE : JUSTIF ICATION DE L'ECRITURE DE J COMME SOMME PONDEREE DE COUT ET DE} $(\mathrm{PROD}-\mathrm{K})^{2}:$

On désire que la production PROD de biomasse soit proche d'une certaine valeur $K$. On peut considêrer cela comme une contrainte : $\mathrm{PROD}=\mathrm{K}$, ce qui revient à minimiser $J=C O U T$ et à introduire $f_{6}=P R O D-K$. Mais les essais numériques sur (A1) et (A2) ne permettent pas d'obtenir une bonne convergence de $u ; J$ tend vers une certaine valeur mais pour $u_{n}$ on observe un phénomène de "zigzag" de ce type :

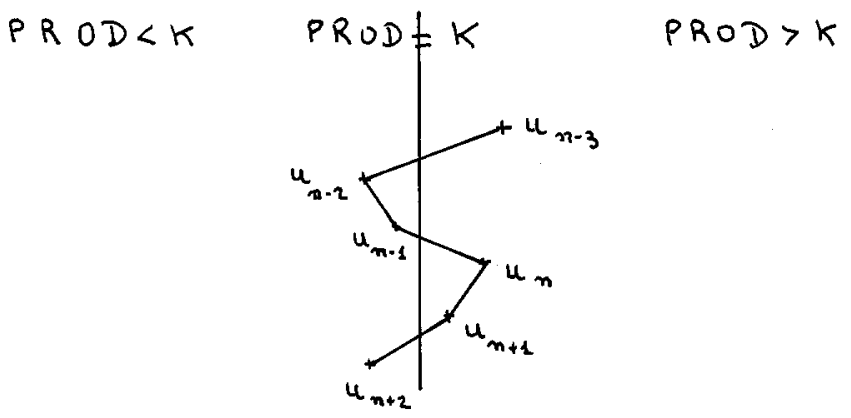

Ce phénomène est lié à la non-convexité et le fait de poser : $J=C O U T+C(P R O D-K)^{2}$ est une sorte de pénalisation de cette contrainte, qui a l'avantage de "convexifier" 
J dans une certaine mesure et d'assurer une bonne convergence des algorithmes.

\section{$\left.3^{\circ}\right)$ Interprëtation biologigue des résultats}

La recherche d'optima locaux à partir de différentes valeurs initiales de u permet de tirer un certain nombre de conclusions sur le système biologique à employer : a) Le fait de s'imposer la production de biomasse nous impose la dimension optimale du fermenteur. En effet, en prenant une production de $20000 \mathrm{~g} / \mathrm{h}$ de biomasse, tous les optima sont tels que le diamètre $F_{1}$ du fermenteur est très voisin de $2 \mathrm{~m}$.

b) Ea concentration $C_{L}$ d'oxygène dissous est très fáible pour les u optimaux, la contrainte $C_{L}>1 \%$ s'avèrant en gẻnéral bloquante. Cela correspond à una minimisation de $\mathrm{K}_{\mathrm{LA}}$ et done du coût du transfert de l'oxygène de la phase gazeuse dans la phase liquide.

c) Le nombre d'aêration NAE, qui peut varier dans I'intervalle $[0,12]$, est proche de 0,2 pour les optima; en corrélation avec lui la vitesse d'aêration $Q$ est petite. Cela correspond à la minimisation du coût de 1'aération. [12]

d) L'introduction d'un recyclage de la biomasse est intéressante, mais ce recyclage n'est pas indispensable quand la concentration $S_{0}$ du substract injecté est grande.

\section{CONCLUSION}

L'optimisation de J nous impose la géométrie du fermenteur, nous montre l'intérêt du recyclage et nous apprend qu'il faut rêduire au minimum indispensable le transfert d'oxygène de la phase gazeuse dans la phase liquide, en diminuant le plus possible l'aêration. Telles sont les premières conclusions qu'on peut tirer de la mise en oeuvre de cette technique d'optimisation des processus de fermentation en continu. Une êtude systêmatique est en cours à l'INRA de Dijon, dans l'idée de mettre en pratique ces algorithmes pour I'optimisation réelle des processus de fermentation en continu sur des installations pilotes et plus tard industrielles. 
1. P.PERINGER, H. BLACHERE,G.CORRIEU et A.G.LANE : "Mathematical model of the kinetics of growth of Saccharomyces Gerivisiae 4th Int. Ferment. Symp., Kyoto, Japan,1972.

2. P.PER INGER, H. BALCHERE,G.CORRIEU et A.G. LANE :"A Generalized Mathematical ModeI for the Growth Kinetics of Saccharomyces Cerivisiae with Experimental Determination of Parameters". Biotechnology and Bioengineering, Vo1. XVI,1974.

3. J. BLUM,P.PERINGER, H. BLACHERE : "Optimal Single Cell Protein Production from Yeasts in a Continuous Fermentation Process". 1st Intersectional Congress of the International Association of Microbiological Societies. Sept.74. Tokyo.

4. S.AIBA,A.HUMPHREY,N.MILIIS : Biochemical Engineering Academic Press. 1965.

5. KJHN, TUCKER : "Non 1inear Programming" :Proceedings of the Second Berkeley Symposium on Mathematical Statistics and Probability" University of California Press. 1961.

6. ARROW-HURWICZ-UZAWA : Studies in Linear and Non Linear Programming : Stanford University Press. 1958.

7. R.GLOWINSKI :"Méthodes Itêratives Duales pour 1a minimisation de fonctionnelles convexes". CTME 1971. Edizioni Cremonese. Rome 1973.

8. J.L.LIONS : Some aspects of the Optimal Control of Distributed Parameter Systems: SIAM. Philadelphia. 1972.

9. J.P. YVON : "Application des méthodes duales au contrôle optimal". Cahier de I'IRIA. 1971.

10. D.LEROY : "Méthodes numériques en contrôle optimal". Thèse 3ème cycle.e. Paris 1972.

11. J.CEA : Optimisation. Thêorie et Algorithmes. Dunod 1971.

12. M. OKABE, S.AIBA,M.OKADA : "The modified complex Method as Applied to an Optimization of Aeration and Agitation in Fermentation". J. Ferment. Technol. Vol. 51. $N^{\circ} 8.1973$. 